\title{
Chronic infection and inflammation affect exercise capacity in cystic fibrosis
}

\author{
P.B. van de Weert-van Leeuwen*, M.G. Slieker*, H.J. Hulzebos", \\ C.L.J.J. Kruitwagen ", C.K. van der Ent* and H.G.M. Arets*
}

ABSTRACT: Pulmonary function and nutritional status are important determinants of exercise capacity in patients with cystic fibrosis (CF). Studies investigating the effects of determinants, such as genotype or infection and inflammation, are scarce and have never been analysed in a multivariate longitudinal model.

A prospective longitudinal cohort study was performed to evaluate whether genotype, chronic inflammation and infection were associated with changes in exercise capacity. Furthermore, we investigated whether exercise capacity can predict clinical outcome.

504 exercise tests of 149 adolescents with CF were evaluated. Maximal oxygen uptake corrected for body mass $\%$ predicted declined $20 \%$ during adolescence, and was associated with immunoglobulin (Ig)G levels and chronic Pseudomonas aeruginosa infection. A lower exercise capacity was associated with a higher mortality, steeper decline in pulmonary function and greater increase in IgG levels.

Since a decline in exercise capacity during adolescence was negatively associated with IgG levels and chronic $P$. aeruginosa infection, these data emphasise the importance of prevention and treatment of chronic inflammation and infections in patients with CF. Furthermore, a lower exercise capacity was associated with a higher mortality rate, steeper decline in pulmonary function and higher increase in IgG levels with increasing age in adolescents with CF. This stresses the value of regular exercise testing for assessing prognosis in adolescents with CF.

KEYWORDS: Aerobic exercise, children, immunoglobulin, immunology, oxygen consumption, respiratory infection

xercise capacity has been identified as an independent predictor of mortality in patients with cystic fibrosis (CF) [1-3]. Regular measurement of maximal oxygen uptake $\left(V^{\prime} \mathrm{O}_{2}\right.$, $\left.\mathrm{max}\right)$ by a maximal cardiopulmonary exercise test (CPET) has, therefore, been emphasised as important in evaluating and assessing prognosis and disease management [4]. Whether exercise capacity can be used as a prognostic marker for clinical outcomes other than mortality rate, such as pulmonary function, chronic inflammation and infection, is unknown. Previous studies showed that pulmonary function [5-10] and nutritional status [5-8] are important determinants of exercise capacity in patients with CF. Studies investigating effects of other potential determinants, such as the cystic fibrosis transmembrane regulator (CFTR) genotype or markers of infection and inflammation, are scarce and have never been analysed in a multivariate longitudinal model.

While the severity of the CFTR mutation (mild versus severe) is associated with pancreatic insufficiency, pulmonary function and survival $[11,12]$, data on the effect of CFTR genotype on exercise capacity are scarce $[13,14]$. It has been shown that CFTR is expressed in the sarcoplasmic reticulum of skeletal muscle $[15,16]$. Deficiency of CFTR protein leads to elevated intracellular calcium levels and enhanced expression of inflammatory genes, which predisposes to muscle wasting in mice [15]. CFTR genotype might, therefore, be a potential determinant of changes in exercise capacity in patients with $\mathrm{CF}$.

CF is a chronic inflammatory disease characterised by the recruitment of high numbers of neutrophils into the infected lungs and the excessive production of immunomodulatory polypeptides. Increased levels of the pro-inflammatory cytokines interleukin (IL)-1 $\beta$, IL-6, tumour necrosis factor- $\alpha$ and IL- 8 have been detected, whereas levels of the anti-inflammatory cytokine IL-10 are decreased [17]. A study in adult patients with $\mathrm{CF}$ showed that higher Creactive protein $(\mathrm{CRP})$, total and specific immunoglobulin (Ig)G, and total leukocyte levels were
AFFILIATIONS

*Dept of Paediatric Pulmonology,

${ }^{*}$ Child Development and Exercise

Centre, Cystic Fibrosis Centre, and

"Dept of Biostatistics, Julius Centre, University Medical Centre Utrecht,

Utrecht, The Netherlands.

\section{CORRESPONDENCE}

P.B. van de Weert-van Leeuwen Dept of Paediatric Pulmonology Cystic Fibrosis Centre University Medical Centre Utrecht Wilhelmina Children's Hospital P0 Box 85090

3508 AB Utrecht The Netherlands E-mail: p.b.vanleeuwen-3@ umcutrecht.nl

Received:

May 202011 Accepted after revision: Aug 042011

First published online: Sept 012011 
significantly associated with decreased pulmonary function $[18,19]$. Another study showed that a change in CRP level was negatively associated with exercise capacity, but the effect of CRP level on exercise capacity was never investigated in a multivariate model [20].

Chronic infections with Pseudomonas aeruginosa are associated with a decline in pulmonary function and increased morbidity and mortality [21, 22], and are known to be an important inducer of hyperinflammation in patients with CF [17]. However, the independent effects of inflammation and chronic $P$. aeruginosa infection on exercise capacity in patients with $\mathrm{CF}$ have never been studied.

The aim of this study was to evaluate whether CFTR genotype, chronic $P$. aeruginosa infection and inflammatory markers, such as total IgG, leukocyte and neutrophil levels, are independently associated with longitudinal changes in exercise capacity in adolescents with CF. Furthermore, we investigated whether exercise capacity can be used as a prognostic marker to predict mortality and chronic $P$. aeruginosa infection rate, changes in inflammatory markers, pulmonary function and body mass index (BMI), in adolescents with CF.

\section{MATERIALS AND METHODS Study design and subjects}

A prospective longitudinal cohort study involving adolescent patients with CF was performed. Patients of the Cystic Fibrosis Centre, University Medical Centre Utrecht (Utrecht, the Netherlands) attended a multidisciplinary examination annually. Pulmonary function tests, CPETs and measurements of inflammation and anthropometrics were routinely performed. Results were prospectively recorded in a database between 1998 and 2008. Sputum cultures were taken during the entire year preceding the annual examination and were retrospectively evaluated and also recorded in the database. Additionally, the database contained information about demographics, decease dates and CFTR genotype.

Patients were included when CPET data from at least two multidisciplinary examinations were available. The database contained longitudinal data of 171 adolescents with $\mathrm{CF}$, who performed 567 CPETs. Patients were 12-18 yrs of age (96 males and 75 females) and were free of pulmonary exacerbations at the time of testing. All patients gave written informed consent for storage and use of their data for scientific purposes, and use of the database was permitted by the ethical board of the University Medical Centre Utrecht. All researchers had full access to all the data in the study and took responsibility for the integrity of the data and the accuracy of the data analysis.

The CPET was performed using an electronically braked cycle ergometer (Ergoline; Cardinal Health, Houten, the Netherlands) according to the protocol of GODFREY [23]. Patients breathed through a face mask (Hans Rudolph Inc., Shawnee, KS, USA), which was connected to a calibrated metabolic cart (Oxycon Pro; Cardinal Health). Volume measurements and breath-by-breath respiratory gas analyses were performed with a flow meter (Triple ${ } \mathrm{V}$ volume transducer; CORTEX Biophysik GmbH, Leipzig, Germany) and gas analyser for oxygen and carbon dioxide (Oxycon Pro). Oxygen consumption, carbon dioxide output and the respiratory exchange ratio (RER) were calculated by a computer from conventional equations. Cardiac frequency $(f c)$ and oxygen saturation were measured continuously during the CPET with a three-lead ECG and a pulse oximeter, respectively. CPET results were only included for analysis when the test was performed until maximal exhaustion. Effort was considered to be maximal when one of the following criteria was met: 1) peak fC $>180$ beats $\cdot \mathrm{min}^{-1}$; 2) peak RER $>1.00$ [24]. Maximal exercise capacity was calculated as the average value over the final $30 \mathrm{~s}$ of the test and was expressed as $V^{\prime} \mathrm{O}_{2}, \max \left(\right.$ in $\left.\mathrm{L} \cdot \mathrm{min}^{-1}\right)$ and $V^{\prime} \mathrm{O}_{2}$ max corrected for body mass $\left(V^{\prime} \mathrm{O}_{2}, \max / \mathrm{kg}\right)$ (in $\left.\mathrm{mL} \cdot \mathrm{kg}^{-1} \cdot \mathrm{min}^{-1}\right)$. For the analysis, maximal exercise values were expressed as \% predicted using reference values for Dutch healthy adolescents, which allowed us to adjust for age, sex and weight. Except for pre-exercise pulmonary function testing, which was not performed in the healthy Dutch adolescents, exercise test criteria were comparable to the protocol used in the patients with CF to estimate maximal exercise capacity and achieve maximal effort [25].

CFTR mutations were divided into five classes, based on the functional effect of the milder of the two mutations. Class I, II and III mutations were categorised as severe, and class IV and $\mathrm{V}$ as mild. If one or both of the mutations were unknown, the patient was classified as unknown [12].

Nutritional status was expressed as the standard deviation score (SDS) for body mass, height and BMI (SDSBMI), based on reference values for healthy Dutch adolescents [26].

Pulmonary function tests were performed prior to the CPET after inhalation of $800 \mu \mathrm{g}$ salbutamol. The forced expiratory volume in $1 \mathrm{~s}$ (FEV1) (in L) was obtained from the best of three maximal expiratory flow-volume curves (Masterscreen; Cardinal Health) and expressed as FEV1 \% pred [27]. All curves were checked for accuracy and repeatability [28].

CRP, total IgG and leukocyte levels were measured in peripheral blood as potential inflammatory determinants of longitudinal changes in exercise capacity. These inflammatory markers were chosen based on the literature concerning chronic inflammation in patients with CF [17] and previous studies showing significant correlations between these specific inflammatory markers and pulmonary function or exercise capacity $[18,19,29]$.

$P$. aeruginosa infection status and decease dates were retrospectively evaluated and recorded in the database. Chronic $P$. aeruginosa infection was considered to be present when $>50 \%$ of the sputa or cough swab cultures were positive in the preceding year [30]. In the older children, fewer than four sputum samples per year were available. In these patients, the old European consensus definition for chronic P. aeruginosa infection was used, i.e. at least three positive cultures over $\geqslant 6$ months with a $\geqslant 1$ month interval [31]. Mortality was defined as death or date of lung transplantation, since these patients are expected to die without transplantation.

\section{Statistical analysis}

A Kolmogorov-Smirnov test was used to test whether the variables were normally distributed. Patient characteristics are presented as mean $\pm \mathrm{SD}$ when variables were normally distributed, otherwise as median (interquartile range). Categorical data are presented as percentages. To evaluate whether CFTR 
genotype, chronic $P$. aeruginosa infection and inflammation were associated with longitudinal changes in exercise capacity in adolescents with CF, a multivariate linear mixed model (LMM) analysis for repeated measurements was used [32]. This statistical technique has several advantages: 1) subjects with missing data are not dropped from the analysis; and 2) within-subject changes and standard errors over time are calculated. Therefore, large variations at the population level, between individuals, do not influence the analysis. This allows us to estimate longitudinal changes over time more accurately compared with a cross-sectional analysis of the data.

Age, FEV1 \% pred, CFTR genotype, chronic P. aeruginosa infection, CRP, total IgG, leukocyte levels, and the interaction terms "IgG levels $\times$ chronic $P$. aeruginosa infection", "FEV1\% pred $\times$ chronic $P$. aeruginosa infection", "CFTR genotype $\times P$. aeruginosa infection" and "age $\times P$. aeruginosa infection" were included as potential independent determinants. The outcome variable was expressed as $V^{\prime} \mathrm{O}_{2}, \mathrm{max} / \mathrm{kg} \%$ pred [25] to adapt for sex-, growth- and maturation-related differences that exist between adolescents of different ages.

In order to investigate whether exercise capacity can predict clinical outcome, exercise capacity measured at the first visit was used to define low $\left(V^{\prime} \mathrm{O}_{2}, \max / \mathrm{kg} \leqslant 80 \%\right.$ pred $)$ and high $\left(V^{\prime} \mathrm{O}_{2}, \mathrm{max} / \mathrm{kg}>80 \%\right.$ pred) exercise capacity groups. The LMM analysis for repeated measurements was used to assess whether this variable was associated with longitudinal changes in pulmonary function, IgG levels or SDSBMI. This was tested by examining the improvement in model fit after the addition of an interaction term to the model (age $\times$ exercise capacity group). The interaction term would allow for different slopes over time for both exercise capacity groups. A log-rank test was used to examine whether there was a difference in chronic $P$. aeruginosa infection or mortality rate between the exercise capacity groups.

All data were analysed in SPSS 18.0 for Windows (SPSS Inc., Chicago, IL, USA). A p-value of $<0.05$ was considered statistically significant. The LMM model was fitted using the Akaike information criterion.

\section{RESULTS}

Data for 504 CPETs of 149 patients (85 males and 64 females) were eligible for inclusion. Mean \pm SD duration of follow-up was $2.8 \pm 1.7$ yrs. Table 1 summarises the baseline characteristics of the patients at the first visit.

Longitudinal changes in $V^{\prime} \mathrm{O}_{2}, \max / \mathrm{kg} \%$ pred were negatively associated with chronic $P$. aeruginosa infection and total IgG levels, but not with CFTR genotype, CRP, total leukocyte levels and/or the interaction terms "chronic $P$. aeruginosa infection $\times$ IgG levels", "chronic P. aeruginosa infection $\times$ FEV1 \% pred", "CFTR genotype $\times P$. aeruginosa infection" and "age $\times P$. aeruginosa infection" (table 2). A longitudinal annual decline of $3.23 \%$ pred was seen in $V^{\prime} \mathrm{O}_{2}, \mathrm{max} / \mathrm{kg}$, independent of chronic $P$. aeruginosa infection and total IgG levels. An additional decline of $4.60 \%$ pred in $V^{\prime} \mathrm{O}_{2}$, max $/ \mathrm{kg}$ was seen when patients became colonised with $P$. aeruginosa $(p=0.007)$. An increase of $1 \mathrm{~g} \cdot \mathrm{L}^{-1}$ in total $\mathrm{IgG}$ levels was associated with a decline in $V^{\prime} \mathrm{O}_{2}$, $\max / \mathrm{kg}$ of $0.54 \%$ pred $(\mathrm{p}=0.020)$. According to the final multivariate mixed model analysis, $V^{\prime} \mathrm{O}_{2}, \mathrm{max} / \mathrm{kg}$ of patients with $\mathrm{CF}$ at the age of 12 yrs is comparable to that of age-matched

\section{TABLE 1 Patient characteristics}

\begin{tabular}{|c|c|}
\hline Patients n & 149 \\
\hline CPETs n & 504 \\
\hline Males \% & 57.1 \\
\hline \multicolumn{2}{|l|}{ CFTR genotype classes $\%$} \\
\hline Class I-III (severe) & 83.2 \\
\hline Class IV-V (mild) & 10.1 \\
\hline Unknown & 6.7 \\
\hline Age yrs & $13.29 \pm 1.24$ \\
\hline Body mass ${ }^{\#}$ & $-0.83 \pm 0.93$ \\
\hline Height $^{\#}$ & $-0.75 \pm 1.00$ \\
\hline $\mathrm{BMI} \mathrm{I}^{\#}$ & $-0.59 \pm 0.97$ \\
\hline FEV $1 \%$ pred & $83.23 \pm 18.04$ \\
\hline Chronic Pseudomonas aeruginosa infection \% & 43.62 \\
\hline Total IgG g. $\mathbf{L}^{-1}$ & $12.39(3.94)$ \\
\hline CRP $\mathrm{mg} \cdot \mathrm{L}^{-1}$ & $6.77(7.49)$ \\
\hline Total leukocytes $\times 10^{9}$ cells $\cdot \mathrm{L}^{-1}$ & $9.31(3.58)$ \\
\hline$V^{\prime} O_{2}, \max L \cdot \min ^{-1}$ & $1.76 \pm 0.49$ \\
\hline$V^{\prime} \mathrm{O}_{2}, \max \%$ pred & $91.66 \pm 28.57$ \\
\hline$V^{\prime} \mathrm{O}_{2}, \max / \mathrm{kg} \mathrm{mL} \cdot \mathrm{kg}^{-1} \cdot \mathrm{min}^{-1}$ & $41.46 \pm 8.89$ \\
\hline$V^{\prime} \mathrm{O}_{2}, \max / \mathrm{kg} \%$ pred & $96.20 \pm 18.29$ \\
\hline$W \max W$ & $142.41 \pm 38.16$ \\
\hline$W_{\max } \%$ pred & $74.75 \pm 20.72$ \\
\hline
\end{tabular}

Data are presented as mean \pm SD or median (interquartile range), unless otherwise stated. CPET: cardiopulmonary exercise test; CFTR: cystic fibrosis transmembrane conductance regulator; BMI: body mass index; FEV1: forced expiratory volume in $1 \mathrm{~s}$; \% pred: \% predicted; Ig: immunoglobulin; CRP: Creactive protein; $V^{\prime} \mathrm{O}_{2}$,max: maximal oxygen uptake; $V^{\prime} \mathrm{O}_{2}$,max/kg: $V^{\prime} \mathrm{O}_{2}$, max corrected for body mass; Wmax: maximal workload. ${ }^{*}$ : standard deviation score for age.

healthy controls (100\%); however, it declines by $20 \%$ pred during adolescence (fig. 1).

Patients with a low $V^{\prime} \mathrm{O}_{2}, \max / \mathrm{kg} \%$ pred at their first visit had a significantly steeper decline in FEV1 \% pred and a higher increase in total IgG levels during follow-up, compared with patients with a high $V^{\prime} \mathrm{O}_{2}$, max/kg \% pred, which was independent of age and body mass. SDSBMI was not associated (table 3). Furthermore, a lower $V^{\prime} \mathrm{O}_{2}, \max / \mathrm{kg} \%$ pred at the first visit was associated with a higher mortality rate during followup of $96.3 \%$ versus $80.0 \%$ (log-rank test, $\mathrm{p}=0.018$ ), but not with chronic $P$. aeruginosa infection rate (log-rank test, $\mathrm{p}=0.903)$.

\section{DISCUSSION}

This study showed that the exercise capacity of adolescents with CF at the age of $12 \mathrm{yrs}$ is comparable to that of agematched healthy controls; however, it declines by $20 \%$ during adolescence. This longitudinal decline in exercise capacity was negatively associated with chronic $P$. aeruginosa infection and IgG levels, independent of age, pulmonary function and body mass. CFTR genotype, CRP, leukocyte and neutrophil levels were not associated. Furthermore, this study showed that clinical outcome can be predicted by exercise capacity. A lower exercise capacity was associated with a higher mortality rate, a steeper decline in pulmonary function and a higher increase in IgG levels during follow-up, but not with SDSBMI and $P$. aeruginosa colonisation rate. 


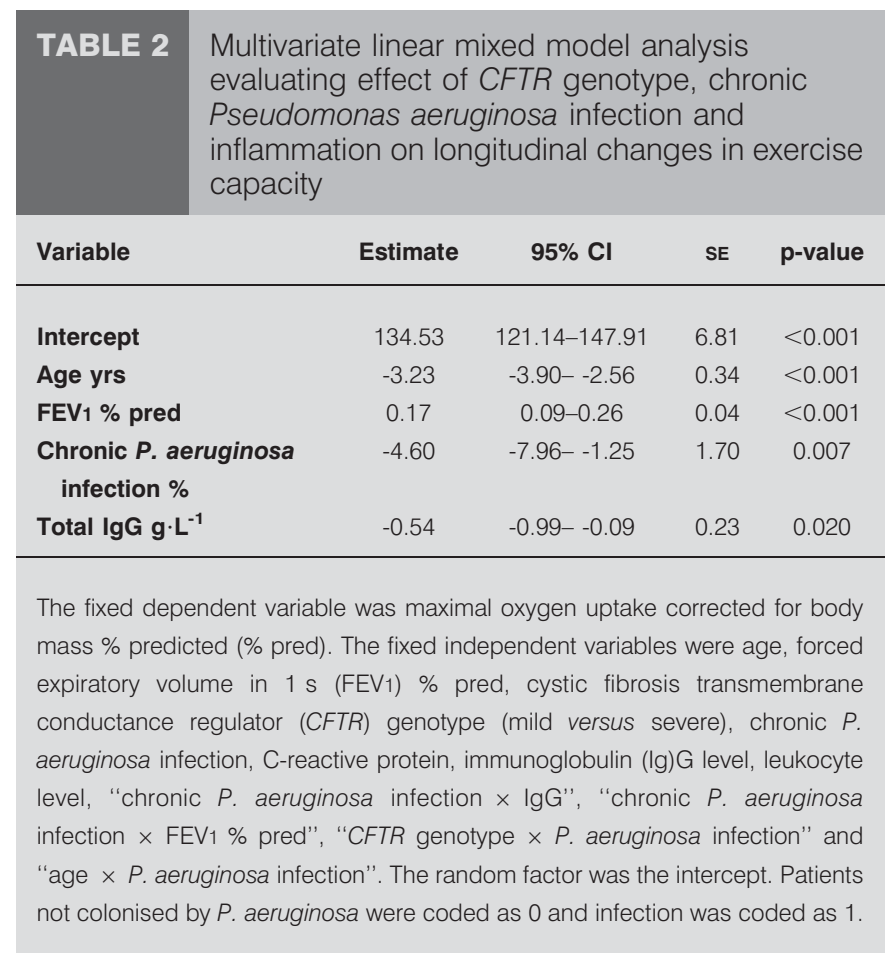

Inverse correlations between elevated IgG levels and pulmonary function have been reported previously in patients with CF [18, 19]. IgG serves to neutralise pathogens such as $P$. aeruginosa and promote its elimination by phagocytes. Elevated $P$. aeruginosa-specific IgG levels can be measured before the organism can be isolated from sputum in patients with CF [33]. Elevated IgG levels might, therefore, be a good representative of inflammation and probably also of $P$. aeruginosa infection status. However, in our model, chronic $P$. aeruginosa infection and IgG levels did not interact, and were independently associated with longitudinal changes in exercise capacity. A possible explanation could be that total IgG levels are rather nonspecific for an infection with $P$. aeruginosa, as it serves as an antibody for infections in general. $P$. aeruginosa-specific IgG levels might interact with $P$. aeruginosa infection status. Unfortunately, we did not measure specific $P$. aeruginosa IgG antibodies.

The negative association between inflammation and exercise capacity is compatible with the concept that chronic systemic inflammation results in a reduced exercise capacity due to devastating effects on skeletal muscle [32, 34], which is not limited to CF disease [20] but is also present in other chronic inflammatory diseases, such as chronic obstructive pulmonary disease [29]. Skeletal muscle mass and function have been shown to be related to exercise capacity in CF [5, 35], but were unfortunately not measured in our study. Furthermore, chronic infection and inflammation may lead to increased rates of intravenous treatment and hospitalisation, which invalidate patients and lead to a reduction in habitual physical activity levels. Although the effect is relatively weak, habitual physical activity levels have been shown to be a significant predictor of $V^{\prime} \mathrm{O}_{2}$, max in patients with $\mathrm{CF}[36,37]$. Recent literature suggests that increasing habitual physical activity levels may have the potential to diminish low-grade inflammation [38]. Whether

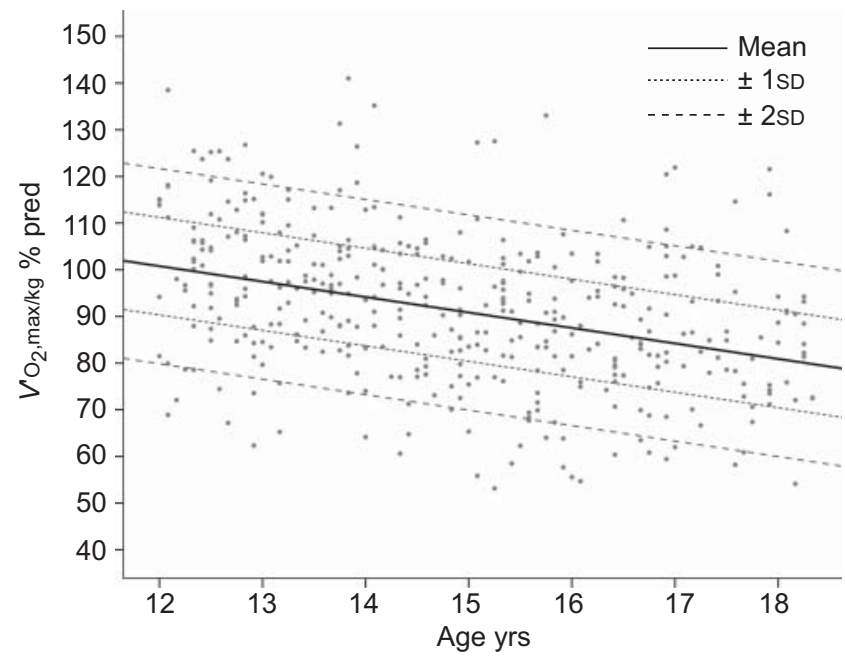

FIGURE 1. Longitudinal decline in maximal oxygen uptake corrected for body mass $\left(V^{\prime} \mathrm{O}_{2}, \mathrm{max} / \mathrm{kg}\right) \%$ predicted (\% pred) during adolescence in patients with cystic fibrosis. Dots represent measured $V^{\prime} \mathrm{O}_{2}$, max $/ \mathrm{kg} \%$ pred. Lines represent calculated $V^{\prime} \mathrm{O}_{2}$, max/kg \% pred based on the final multivariate mixed model analysis.

regular exercise can also diminish inflammation in patients suffering from severe infections and inflammation, such as patients with $\mathrm{CF}$, is unknown. Unfortunately, habitual physical activity levels, rate of intravenous treatment and hospitalisation were not recorded in the database. Moreover, chronic infection and inflammation can lead to a reduced appetite [39] and, therefore, to a reduction in body mass, which may lead to a reduction in exercise capacity. However, this is not the most likely explanation, as exercise capacity was adjusted for differences in body mass in our model.

Although CRP levels were found to be negatively correlated with exercise capacity in patients with CF in a univariate model [20], we did not find an association between CRP levels and longitudinal changes in exercise capacity. CRP is an acute phase reactant that is predominantly elevated during acute infectious diseases and only slightly in patients suffering from chronic infectious diseases. IgG levels might be a better representative of chronic inflammation and, therefore, a better predictor of longitudinal changes in exercise capacity in patients with $\mathrm{CF}$, compared with CRP.

Chronic infection with $P$. aeruginosa was associated with a decline in $V^{\prime} \mathrm{O}_{2}$, max $/ \mathrm{kg}$ of $4.60 \%$ pred, independent of age, IgG levels, pulmonary function and nutritional status. Chronic $P$. aeruginosa infection may have an effect on exercise capacity by weakening the diaphragm. In a mouse model, it was shown that a pulmonary infection with $P$. aeruginosa preferentially weakened the diaphragm, an effect not directly correlated with the degree of pulmonary inflammation [40]. Chronic infections with $P$. aeruginosa were found to be associated with other clinical variables as well, such as a decline in pulmonary function and increased morbidity and mortality [21, 22].

We did not find an association between genotype and $V^{\prime} \mathrm{O}_{2}, \max / \mathrm{kg} \%$ pred in our study, which is consistent with the results of KAPLAN et al. [13], but in contrast with the findings of SELVADURAI et al. [14], who showed that patients with a mild mutation (class IV and V) had a better exercise capacity than 
TABLE 3 Multivariate linear mixed model analysis evaluating whether exercise capacity can predict longitudinal changes in pulmonary function, total IgG levels or SDSBMI

\begin{tabular}{|c|c|c|c|c|c|}
\hline Dependent variable & Variable & Estimate & $95 \% \mathrm{Cl}$ & $\mathrm{SE}$ & p-value \\
\hline \multirow[t]{3}{*}{ FEV $1 \%$ pred } & Intercept & 89.83 & $76.30-103.36$ & 6.87 & $<0.001$ \\
\hline & Age & -1.35 & $-2.29--0.41$ & 0.48 & 0.005 \\
\hline & Exercise capacity group $\times$ age & 1.03 & $0.53-1.54$ & 0.26 & $<0.001$ \\
\hline \multirow[t]{2}{*}{$\lg \mathbf{G} \cdot \mathrm{L}^{-1}$} & Intercept & 3.98 & $4.31-9.64$ & 1.35 & $<0.001$ \\
\hline & Age & 0.52 & $0.33-0.71$ & 0.10 & $<0.001$ \\
\hline \multirow{2}{*}{ SDSBмı } & Age & 0.05 & $0.00-0.09$ & 0.02 & 0.030 \\
\hline & Exercise capacity group $\times$ age & 0.02 & $-0.01-0.05$ & 0.01 & 0.062 \\
\hline
\end{tabular}

The fixed dependent variables were forced expiratory volume in $1 \mathrm{~s}(\mathrm{FEV} 1)$ \% predicted (\% pred), immunoglobulin (Ig)G level or standard deviation score for body mass index (SDSBMI). The fixed independent variables were age and "exercise capacity group $\times$ age". The random factor was the intercept. Exercise capacity was coded as 0 or low when maximal oxygen uptake corrected for body mass $\left(V^{\prime} \mathrm{O}_{2}, \max / \mathrm{kg}\right) \leqslant 80 \%$ pred, or as 1 or high when $V^{\prime} \mathrm{O}_{2}$,max $/ \mathrm{kg}>80 \%$ pred.

patients with a severe mutation (class I-III). KAPLAN et al. [13] compared two groups of patients who were either homozygous $(n=10)$ or heterozygous $(n=20)$ for the $\Delta F 508$ mutation. The study was limited by its small sample size. Additionally, all heterozygous patients were pooled in one group, independent of the classification of the second mutation. The study of SELVADURAi et al. [14] was limited because the effect of CFTR genotype on exercise capacity was only univariately examined. Therefore, analysing the effect of CFTR genotype on exercise capacity in a longitudinal multivariate model adds to our current knowledge. Moreover, identification of genetic modifiers might be of importance in elucidating the association between genotypic and phenotypic differences among patients.

Furthermore, our longitudinal study showed that exercise capacity can be used as a prognostic marker for clinical outcome. The value of the CPET in predicting survival has been evaluated previously. Consistent with our results, these studies showed that exercise capacity was associated with mortality rate [1-3]. Life expectancy in CF patients has largely improved in the last few decades, but it is still reduced compared with the healthy population. Additionally, the clinical course differs greatly between patients. Therefore, determining prognosis is an important issue in disease management. A number of variables, such as pulmonary function [1,41], sex [42], nutritional status [41] and chronic infection with $P$. aeruginosa [21, 22], have been related to prognosis. Exercise capacity is an important addition to these predictors because it represents a patient's functional capacity. We are the first to show that exercise capacity can also predict changes in pulmonary function and inflammation with increasing age in adolescents with CF. Exercise capacity measured at the first visit was not associated with differences in chronic $P$. aeruginosa infection rate. Many patients were already colonised with $P$. aeruginosa at the start of the study, which reduced the $a$ priori chance of a new colonisation event.

The longitudinal cohort design is one of the key strengths of this study. Longitudinal changes can be estimated more accurately as subject-specific changes over time can be estimated. However, a few limitations should be considered.
We did not analyse all potential confounders, such as habitual physical activity levels, muscle mass and/or function and effects of other inflammatory markers, such as IL-6. IL-6 is known to be involved in exercise metabolism [43], but is unfortunately not routinely measured.

In conclusion, this longitudinal cohort study showed that the exercise capacity of patients with CF declines during adolescence compared with age-matched healthy controls, which is negatively associated with total $\operatorname{IgG}$ levels and chronic infection with $P$. aeruginosa. Since a negative association was observed between exercise capacity and markers of chronic inflammation and infection, these findings emphasise the importance of prevention and aggressive treatment of chronic inflammation and infections in adolescents with CF. Furthermore, a lower exercise capacity was associated with a higher mortality rate, a steeper decline in pulmonary function and a greater increase in total $\mathrm{IgG}$ levels with increasing age in adolescents with CF. This stresses the significance of regular CPETs for assessing the prognosis of adolescents with CF.

\section{SUPPORT STATEMENT}

This study was supported by an unrestricted grant from the Dutch Cystic Fibrosis Society.

\section{STATEMENT OF INTEREST}

A statement of interest for C.K. van der Ent can be found at www.erj. ersjournals.com/site/misc/statements.xhtml

\section{ACKNOWLEDGEMENTS}

The authors would like to thank the lung function technicians of the Dept of Paediatric Pulmonology (Cystic Fibrosis Centre, University Medical Centre Utrecht, Utrecht, the Netherlands) for collecting the data.

\section{REFERENCES}

1 Nixon PA, Orenstein DM. The prognostic value of exercise testing in patients with cystic fibrosis. N Engl J Med 1992; 327: 1785-1788.

2 Moorcroft AJ, Dodd ME, Webb AK. Exercise testing and prognosis in adult cystic fibrosis. Thorax 1997; 52: 291-293. 
3 Pianosi P, Leblanc J, Almudevar A. Peak oxygen uptake and mortality in children with cystic fibrosis. Thorax 2005; 60: 50-54.

4 Clinical exercise testing with reference to lung diseases: indications, standardization and interpretation strategies. ERS Task Force on Standardization of Clinical Exercise Testing. Eur Respir J 1997; 10: 2662-2689.

5 de Meer K, Gulmans VAM, Van der Laag J. Peripheral muscle weakness and exercise capacity in children with cystic fibrosis. Am J Respir Crit Care Med 1999; 159: 748-754.

6 Klijn PH, Van der Net J, Kimpen JL, et al. Longitudinal determinants of peak aerobic performance in children with cystic fibrosis. Chest 2003; 124: 2215-2219.

7 Lands LC, Heigenhauser GJ, Jones NL. Analysis of factors limiting maximal exercise performance in cystic fibrosis. Clin Sci (Colch) 1992; 83: 391-397.

8 Moorcroft AJ, Dodd ME, Morris J, et al. Symptoms, lactate and exercise limitation at peak cycle ergometry in adults with cystic fibrosis. Eur Respir J 2005; 25: 1050-1056.

9 Pianosi P, Leblanc J, Almudevar A. Relationship between FEV1 and peak oxygen uptake in children with cystic fibrosis. Pediatr Pulmonol 2005; 40: 324-329.

10 Shah AR, Gozal D, Keens TG, et al. Determinants of aerobic and anaerobic exercise performance in cystic fibrosis. Am J Respir Crit Care Med 1998; 157: 1145-1150.

11 McKone EF, Emerson SS, Edwards KL, et al. Effect of genotype on phenotype and mortality in cystic fibrosis: a retrospective cohort study. Lancet 2003; 361: 1671-1676.

12 McKone EF, Goss CH, Aitken ML. CFTR genotype as a predictor of prognosis in cystic fibrosis. Chest 2006; 130: 1441-1447.

13 Kaplan TA, Moccia-Loos G, Rabin M, et al. Lack of effect of delta F508 mutation on aerobic capacity in patients with cystic fibrosis. Clin J Sport Med 1996; 6: 226-231.

14 Selvadurai $\mathrm{HC}$, McKay $\mathrm{KO}$, Blimkie CJ, et al. The relationship between genotype and exercise tolerance in children with cystic fibrosis. Am J Respir Crit Care Med 2002; 165: 762-765.

15 Divangahi M, Balghi H, Danialou G, et al. Lack of CFTR in skeletal muscle predisposes to muscle wasting and diaphragm muscle pump failure in cystic fibrosis mice. PLoS Genet 2009; 5: e1000586.

16 Lamhonwah AM, Bear CE, Huan LJ, et al. Cystic fibrosis transmembrane conductance regulator in human muscle: dysfunction causes abnormal metabolic recovery in exercise. Ann Neurol 2010; 67: 802-808.

17 De Rose V. Mechanisms and markers of airway inflammation in cystic fibrosis. Eur Respir J 2002; 19: 333-340.

18 Levy H, Kalish LA, Huntington I, et al. Inflammatory markers of lung disease in adult patients with cystic fibrosis. Pediatr Pulmonol 2007; 42: 256-262.

19 Cowan RG, Winnie GB. Anti-Pseudomonas aeruginosa IgG subclass titers in patients with cystic fibrosis: correlations with pulmonary function, neutrophil chemotaxis, and phagocytosis. J Clin Immunol 1993; 13: 359-370.

20 Bradley J, McAlister O, Elborn S. Pulmonary function, inflammation, exercise capacity and quality of life in cystic fibrosis. Eur Respir J 2001; 17: 712-715.

21 Emerson J, Rosenfeld M, McNamara S, et al. Pseudomonas aeruginosa and other predictors of mortality and morbidity in young children with cystic fibrosis. Pediatr Pulmonol 2002; 34: 91-100.

22 Kosorok MR, Zeng L, West SE, et al. Acceleration of lung disease in children with cystic fibrosis after Pseudomonas aeruginosa acquisition. Pediatr Pulmonol 2001; 32: 277-287.

23 Godfrey S. Exercise Testing in Children. Philadelphia, WB Saunders, 1974.

24 Nixon PA, Orenstein DM. Exercise testing in children. Pediatr Pulmonol 1988; 5: 107-122.

25 Binkhorst RA, van 't Hof MA, Saris WHM. Maximum exercise in children: reference-values for 6-18 years old girls and boys. The Hague, Dutch Heart Association, 1992.

26 TNO Prevention and Health. Growth Diagrams 1997. Houten, Bohn Stafleu Van Loghum, 1998.

27 Zapletal A, Samenek TP. Lung function in children and adolescents: methods and reference values. Basel, Karger, 1987.

28 Miller MR, Hankinson J, Brusasco V, et al. Standardisation of spirometry. Eur Respir J 2005; 26: 319-338.

29 Broekhuizen R, Wouters EF, Creutzberg EC, et al. Raised CRP levels mark metabolic and functional impairment in advanced COPD. Thorax 2006; 61: 17-22.

30 Lee TW, Brownlee KG, Conway SP, et al. Evaluation of a new definition for chronic Pseudomonas aeruginosa infection in cystic fibrosis patients. J Cyst Fibros 2003; 2: 29-34.

31 Doring G, Conway SP, Heijerman HG, et al. Antibiotic therapy against Pseudomonas aeruginosa in cystic fibrosis: a European consensus. Eur Respir J 2000; 16: 749-767.

32 Tramper-Stranders GA, Van der Ent CK, Wolfs TF. Detection of Pseudomonas aeruginosa in patients with cystic fibrosis. J Cyst Fibros 2005; 4: Suppl. 2, 37-43.

33 Coletti D, Moresi V, Adamo S, et al. Tumor necrosis factor- $\alpha$ gene transfer induces cachexia and inhibits muscle regeneration. Genesis 2005; 43: 120-128.

34 Haddad F, Zaldivar F, Cooper DM, et al. IL-6-induced skeletal muscle atrophy. J Appl Physiol 2005; 98: 911-917.

35 Moser C, Tirakitsoontorn P, Nussbaum E, et al. Muscle size and cardiorespiratory response to exercise in cystic fibrosis. Am J Respir Crit Care Med 2000; 162: 1823-1827.

36 Hebestreit H, Kieser S, Rüdiger S, et al. Physical activity is independently related to aerobic capacity in cystic fibrosis. Eur Respir J 2006; 28: 734-739.

37 Troosters T, Langer D, Vrijsen B, et al. Skeletal muscle weakness, exercise tolerance and physical activity in adults with cystic fibrosis. Eur Respir J 2009; 33: 99-106.

38 Handschin C, Spiegelman BM. The role of exercise and PGC1 $\alpha$ in inflammation and chronic disease. Nature 2008; 454: 463-469.

39 Nasr SZ, Drury D. Appetite stimulants use in cystic fibrosis. Pediatr Pulmonol 2008; 43: 209-219.

40 Divangahi M, Matecki S, Dudley RW, et al. Preferential diaphragmatic weakness during sustained Pseudomonas aeruginosa lung infection. Am J Respir Crit Care Med 2004; 169: 679-686.

41 Kerem E, Reisman J, Corey M, et al. Prediction of mortality in patients with cystic fibrosis. $N$ Engl J Med 1992; 326: 1187-1191.

42 Rosenfeld M, Davis R, FitzSimmons S, et al. Gender gap in cystic fibrosis mortality. Am J Epidemiol 1997; 145: 794-803.

43 Pedersen BK. The diseasome of physical inactivity - and the role of myokines in muscle-fat cross talk. J Physiol 2009; 587: 5559-5568. 